\title{
Bacterial load and defective monocyte-derived macrophage bacterial phagocytosis in biomass smoke-related COPD
}

\author{
Baishakhi Ghosh ${ }^{1,2}$, Akshay H. Gaike ${ }^{3,4}$, Kanchan Pyasi ${ }^{1}$, Bill Brashier ${ }^{1}$, \\ Vandana V. Das ${ }^{1}$, Jyoti D. Londhe ${ }^{1}$, Sanjay Juvekar ${ }^{4}$, Yogesh S. Shouche ${ }^{3}$, \\ Louise E. Donnelly ${ }^{5}$, Sundeep S. Salvi ${ }^{1}$ and Peter J. Barnes ${ }^{5}$
}

Affiliations: ${ }^{1}$ Chest Research Foundation, Pune, India. ${ }^{2}$ Faculty of Health and Biomedical Sciences, Symbiosis International University, Pune, India. ${ }^{3}$ National Centre for Microbial Resource, National Centre for Cell Sciences, Pune, India. ${ }^{4}$ adu Rural Health Program, KEM Hospital Research Centre, Pune, India. ${ }^{5}$ National Heart and Lung Institute, Imperial College London, London, UK.

Correspondence: Sundeep S. Salvi, Chest Research Foundation, Survey No. 15, Marigold Complex, Kalyani Nagar, Pune 411014, India. E-mail: ssalviđarfindia.com

@ERSpublications

Bacterial load in biomass smoke-exposed COPD subjects is associated with defective macrophage bacterial phagocytosis http://ow.ly/Q5ns30mNN0k

Cite this article as: Ghosh B, Gaike AH, Pyasi K, et al. Bacterial load and defective monocyte-derived macrophage bacterial phagocytosis in biomass smoke-related COPD. Eur Respir J 2019; 53: 1702273 [https://doi.org/10.1183/13993003.02273-2017].

ABSTRACT Lower airway colonisation with species of potentially pathogenic bacteria (PPB) is associated with defective bacterial phagocytosis, in monocyte-derived macrophages (MDMs) and alveolar macrophages, from tobacco smoke-associated chronic obstructive pulmonary disease (S-COPD) subjects. In the developing world, COPD among nonsmokers is largely due to biomass smoke (BMS) exposure; however, little is known about PPB colonisation and its association with impaired innate immunity in these subjects.

We investigated the PPB load (Streptococcus pneumoniae, Haemophilus influenzae, Moraxella catarrhalis and Pseudomonas aeruginosa) in BMS-exposed COPD (BMS-COPD) subjects compared with S-COPD and spirometrically normal subjects. We also examined the association between PPB load and phagocytic activity of MDMs and lung function. Induced sputum and peripheral venous blood samples were collected from 18 healthy nonsmokers, 15 smokers without COPD, 16 BMS-exposed healthy subjects, 19 S-COPD subjects and 23 BMS-COPD subjects. PPB load in induced sputum and MDM phagocytic activity were determined using quantitative PCR and fluorimetry, respectively.

Higher bacterial loads of $S$. pneumoniae, $H$. influenzae and $P$. aeruginosa were observed in BMS-COPD subjects. Increased PPB load in BMS-exposed subjects was significantly negatively associated with defective phagocytosis in MDMs and spirometric lung function indices $(\mathrm{p}<0.05)$.

Increased PPB load in airways of BMS-COPD subjects is inversely associated with defective bacterial phagocytosis and lung function. 


\section{Introduction}

Chronic obstructive pulmonary disease (COPD) is a progressive, debilitating, inflammatory airway condition usually caused by chronic exposure to noxious inhaled pollutants. It is the third leading cause of death worldwide and second leading cause of death in India [1,2]. Tobacco smoking is the most established risk factor for COPD development, although its population-attributable fraction is $<50 \%$ [3]. Recent evidence from developing countries reveals that exposure to smoke generated from the combustion of biomass fuel (i.e. indoor or household air pollution) in poorly ventilated homes is also an important risk factor for COPD, especially in females [3-5]. It has been estimated that $70 \%$ of houses in India and $\sim 50 \%$ of the houses in the world, especially countries in Africa, Southeast Asia, South Asia and South America, use biomass fuel for cooking and heating purposes [5]. About $85 \%$ of COPD in a rural population in India was found among people who had never smoked [4].

The airways of tobacco smoke-associated COPD (S-COPD) subjects are frequently colonised with species of potentially pathogenic bacteria ( $\mathrm{PPB}$ ), whereas the airways of smokers without COPD and healthy nonsmokers are usually not colonised with PPB $[6,7]$. The presence of PPB is strongly associated with recurrent acute exacerbations, increased airway inflammation and progressive decline in lung function in COPD subjects $[8,9]$. Exacerbations are important clinical characteristics of COPD, and are major contributors to morbidity and mortality [10]. PATEL et al. [11] reported that bacterial colonisation increases the frequency of exacerbations in COPD subjects, denoting a role of bacterial load in determining the presence and severity of exacerbations. The commonest pathogenic organisms that colonise the lower respiratory tract among COPD subjects are Haemophilus influenzae, Streptococcus pneumoniae, Moraxella catarrhalis and Pseudomonas aeruginosa $[7,9,11]$. The loads of these pathogenic bacteria were found to be increased during COPD exacerbations, indicating their possible role in causation $[9,11]$.

Macrophages are the key immune cells that play an important role in removal of PPB from the airways [12]. The removal of PPB via phagocytosis is essential for maintaining a pathogen-free airway. However, impaired phagocytic activity of macrophages for bacteria is observed in S-COPD subjects. Macrophages from lungs of S-COPD subjects showed reduced phagocytic activity to $H$. influenzae and S. pneumoniae $[13,14]$. This has been implicated as one of the underlying causes for persistent infection and inflammation in the lungs of COPD patients $[8,15]$.

Obtaining macrophages from lungs to study their phagocytic function requires invasive procedures such as bronchoscopy. Blood monocytes migrate from the circulation to the lungs and differentiate into macrophages, and the population of these macrophages is found to be increased in COPD [16]. Monocyte-derived macrophages (MDMs) are widely used as a model to understand the phagocytic activity of macrophages in vitro in many inflammatory airway diseases, such as COPD, asthma and cystic fibrosis [17-19]. However, we and others have shown that MDMs can be used as a surrogate for lung macrophages $[17,18,20]$. Prior studies in S-COPD subjects have reported that MDMs from S-COPD subjects exhibit defective bacterial phagocytosis compared with healthy subjects and this phagocytic defect does not differ significantly from that in alveolar macrophages [17]. As MDMs are easily and safely accessible, they are widely used to study macrophage biology of the airways [21].

Defective bacterial phagocytosis by MDMs has been shown to be associated with greater colonisation of PPB in the respiratory tract of S-COPD subjects $[17,22]$, although these studies need to be replicated in other parts of world. However, the phagocytic ability of MDMs in biomass smoke (BMS)-exposed COPD (BMS-COPD) subjects has not been reported. In this study, we investigated the airway PPB load and bacterial phagocytic activity of MDMs from BMS-COPD subjects, and compared this with S-COPD and healthy subjects residing in rural India.

\section{Methods}

\section{Study subjects}

We recruited 47 COPD patients (19 S-COPD and 28 BMS-COPD) and 49 controls (18 nonsmokers (H-NS), 15 smokers without COPD (HS) and 16 BMS-exposed healthy subjects (H-BMS)) from the Chest Research Foundation (CRF; Pune, India) COPD cohort (supplementary material). Quality of life was assessed with the COPD Assessment Test (CAT) and St George's Respiratory Questionnaire (SGRQ) for COPD $[23,24]$. The diagnosis of COPD was established using Global Initiative for Chronic Obstructive Lung Disease (GOLD) criteria, i.e. presence of post-bronchodilator forced expiratory volume in $1 \mathrm{~s}$ $\left(\mathrm{FEV}_{1}\right) /$ forced vital capacity $(\mathrm{FVC})<70 \%$ and $\mathrm{FEV}_{1}<80 \%$ pred [25]. Age-matched controls were recruited from the same villages, and had normal spirometry, no underlying clinical abnormality and normal chest radiography.

The criterion of H-BMS phenotype and BMS-COPD diagnosis was based on a minimum of $2 \mathrm{~h}$ of exposure to BMS per day for at least 20 years [26]. The BMS exposure was expressed as hours-year 
(product of the number of years exposed to smoke due to combustion of biomass fuel and the average number of hours spent daily around the biomass fuel smoke). HS and S-COPD subjects were participants who had smoked $\geqslant 10$ pack-years. Subjects with a past or current history of passive smoking, tuberculosis, asthma, lung cancer or allergy were excluded from the study. In addition, the recruited COPD subjects had no exacerbations/clinical infections during enrolment and were not under any systemic/inhaled corticosteroid or antibiotic treatment in the preceding 12 weeks.

Written informed consent was obtained from all subjects and the study was approved by the Institutional Ethics Committee of CRF.

\section{Spirometry}

Spirometry was performed in accordance with European Respiratory Society/American Thoracic Society guidelines [27]. Values were expressed either as absolute volumes or flows, or as percentage predicted based on age, sex, weight, height and ethnicity.

\section{Sputum induction}

Sputum induction was performed by nebulising 3\% (w/v) hypertonic saline through an ultrasonic nebuliser (Omron, Kyoto, Japan) for 15-20 min. Differential cell count was also performed on the induced sputum samples. The detailed protocol of sputum induction and differential cell count is outlined in the supplementary material.

\section{Quantitative PPB culture}

Quantitative bacterial culture was performed in the induced sputum for S. pneumoniae, H. influenzae, M. catarrhalis and $P$. aeruginosa as described in the supplementary material.

\section{DNA extraction from sputum samples}

DNA was extracted from the stored induced sputum samples according to the manufacturer's instructions using a QIAmp DNA Mini Kit (Qiagen, Hilden, Germany) and stored at $-20^{\circ} \mathrm{C}$. DNA quantification was performed using a NanoDrop ND-1000 spectrophotometer (ThermoFisher Scientific, Waltham, MA, USA) and quality was checked on $0.8 \%$ agarose gel.

\section{Standard bacterial DNA}

DNA of S. pneumoniae (ATCC 49619/DSM 24048), H. influenzae (ATCC 10211/DSM 11969) and M. catarrhalis (ATCC 9143/DSM 9143) was purchased from the German Collection of Microorganisms and Cell Cultures (Leibniz Institute DSMZ, Leibniz, Germany). The DNA from P. aeruginosa (ATCC 27853/MCC 2080) was isolated at the Microbial Culture Collection, National Centre for Cell Sciences (Pune, India) using a MoBio Microbial DNA Isolation Kit (Qiagen) according to the manufacturer's instructions.

\section{Quantitative PCR}

Quantitative PCR (qPCR) was performed using the SYBR Green assay to quantify S. pneumoniae, $H$. influenzae, $M$. catarrhalis and P. aeruginosa by targeting validated primers (Spn9802, P4 lipoprotein, $\mathrm{CopB}$ and $\mathrm{Pa} 23$, respectively) according to previously defined protocols established on sputum samples $[15,28]$. The primer sequences and GPCR program are listed in supplementary tables S1 and S2, respectively.

\section{Monocyte isolation and phagocytosis assay}

Monocytes were isolated from peripheral venous blood and differentiated to MDMs as described in the supplementary material. The phagocytic activity of MDMs to fluorescently labelled polystyrene beads or heat-killed nonopsonised PPB was measured as described previously (supplementary material) [17].

\section{Viability assay}

After phagocytosis, the viability of MDMs was determined by the 3-(4,5-dimethythiazol-2-yl)-2,5diphenyltetrazolium bromide (MTT) assay (supplementary material).

\section{Statistical analysis}

Prism version 7.0 (GraphPad, San Diego, CA, USA) was used for analysis. Normality of data was determined by the Shapiro-Wilk normality test. For nonparametric data, Kruskal-Wallis/Dunn's multiple comparisons test was performed; for parametric data, ANOVA/Tukey's multiple comparisons test was performed. Correlations were determined using the Spearman rank correlation test. SPSS version 23.0 
(IBM, Armonk, NY, USA) was used to perform multivariate linear regression analysis. Differences were considered significant if $\mathrm{p}<0.05$.

\section{Results}

Study subjects

The 91 subjects recruited to the study included $18 \mathrm{H}-\mathrm{NS}, 15 \mathrm{HS}, 16 \mathrm{H}-\mathrm{BMS}, 19 \mathrm{~S}-\mathrm{COPD}$ and 23 BMS-COPD subjects. Out of 91 subjects, only 76 subjects provided usable sputum samples $(\geqslant 0.5 \mathrm{~g}$ sputum plugs per sample) for quantifying the bacterial load (14 H-NS, $11 \mathrm{HS}, 15 \mathrm{H}-\mathrm{BMS}, 16 \mathrm{~S}-\mathrm{COPD}$ and 20 BMS-COPD). The demographic details are presented in table 1 (76 subjects) and supplementary table S3 (91 subjects).

There were no significant differences in age between the study groups. The number of pack-years smoked was similar between HS and S-COPD subjects $(p>0.05)$. Similarly, the BMS exposure history was similar between H-BMS and BMS-COPD subjects $(p>0.05)$. The total CAT score of S-COPD subjects was significantly higher compared with BMS-COPD subjects $(\mathrm{p}<0.05)$, but the total SGRQ score and spirometric indices were not statistically different among the COPD subgroups (S-COPD and BMS-COPD) $(p>0.05)$. Furthermore, there were no statistically significant differences in $\beta$-agonist and anticholinergic use between S-COPD and BMS-COPD subjects.

\section{Load of pathogenic microbes as determined by qPCR}

Initially, a sex-based comparison for PPB was performed by comparing sputum samples from male subjects (H-NS, HS and S-COPD) and female subjects (H-BMS and BMS-COPD). We did not observe any significant difference in the load of each PPB (S. pneumoniae, H. influenzae, M. catarrhalis or P. aeruginosa) and overall

TABLE 1 Demographic characteristics of the 40 healthy and 36 chronic obstructive pulmonary disease (COPD) subjects

\begin{tabular}{|c|c|c|c|c|c|}
\hline & \multicolumn{3}{|c|}{ Healthy subjects } & \multicolumn{2}{|c|}{ COPD subjects } \\
\hline & H-NS & HS & H-BMS & S-COPD & BMS-COPD \\
\hline Subjects & 14 & 11 & 15 & 16 & 20 \\
\hline \multicolumn{6}{|l|}{ Sex } \\
\hline Male & 14 & 11 & 0 & 16 & 0 \\
\hline Female & 0 & 0 & 15 & 0 & 20 \\
\hline Current smoker & 0 & 9 & 0 & 14 & 0 \\
\hline Ex-smoker & 0 & 2 & 0 & 2 & 0 \\
\hline BMS exposure history hours-year & $0(0-0)$ & $0(0-0)$ & $138(130-252)^{\#,, 1, \S}$ & $0(0-0)$ & $134(120-204)^{\#, 9, \S}$ \\
\hline \multicolumn{6}{|l|}{ BMS exposure status } \\
\hline Currently exposed & 0 & 0 & 15 & 0 & 23 \\
\hline Previously exposed & 0 & 0 & 0 & 0 & 0 \\
\hline 2 (moderate) & & & & 5 & 6 \\
\hline 3 (severe) & & & & 7 & 4 \\
\hline 4 (very severe) & & & & 2 & 4 \\
\hline Use of $\beta$-agonists & & & & $43.75(7)$ & $45(9)$ \\
\hline Use of anticholinergics & & & & $12.5(2)$ & $20(4)$ \\
\hline $\mathrm{FEV}_{1} / \mathrm{FVC} \%$ & $78.06 \pm 6.29$ & $77.75 \pm 3.98$ & $79.25 \pm 4.91$ & $53.06 \pm 11.51^{\#, 1,+}$ & $52.96 \pm 9.34^{\#,,_{1++}}$ \\
\hline Post-bronchodilator FEV 1 \% pred & $102.80 \pm 11.35$ & $91.60 \pm 6.52$ & $95.76 \pm 10.76$ & $46.43 \pm 15.12^{\#, 1,+}$ & $50.17 \pm 14.10^{\#, n,+}$ \\
\hline Post-bronchodilator FVC \% pred & 99.81 & 88.77 & 99.37 & 68.72 & 74.27 \\
\hline & (95.23-108.2) & $(85.46-98.81)^{\# .^{+}}$ & (91.38-111.1) & $(57.57-86.38)^{\#, n_{,++}}$ & $(56.86-93.63)^{\#,,_{,+}}$ \\
\hline
\end{tabular}

Data are presented as $n$, mean \pm SD, median (interquartile range) or $\%$ (n). BMS: biomass smoke; H-NS: healthy nonsmokers; HS: smokers without COPD; H-BMS: BMS-exposed healthy subjects; S-COPD: tobacco smoke-associated COPD subjects; BMS-COPD: BMS-exposed COPD subjects; CAT: COPD Assessment Test; SGRQ: St George's Respiratory Questionnaire; GOLD: Global Initiative for Chronic Obstructive Lung Disease; FEV1: forced expiratory volume in $1 \mathrm{~s}$; FVC: forced vital capacity. The Shapiro-Wilk normality test was performed. Within-group comparison was performed by the Kruskal-Wallis/Dunn's multiple comparisons test for nonparametric data and ANOVA/Tukey's multiple comparisons test for parametric data. ${ }^{\#}$ : $p \leqslant 0.05$ versus $H-N S$; ${ }^{\uparrow}$ : $p \leqslant 0.05$ versus $\mathrm{HS}^{+}{ }^{+}: \mathrm{p} \leqslant 0.05$ versus $\mathrm{H}-\mathrm{BMS}$; ${ }^{\S}$ : $p \leqslant 0.05$ versus $\mathrm{S}-\mathrm{COPD}$. 
PPB load (mean load of S. pneumoniae, H. influenzae, M. catarrhalis and P. aeruginosa) in the airways between the male and female subjects ( $\mathrm{p}>0.05)$ (supplementary figures S1 and S2).

For the induced sputum qPCR assay of $S$. pneumoniae and P. aeruginosa, a cut-off value of $>1 \times 10^{4}$ genomic copies $\cdot \mathrm{mL}^{-1}$ was considered $[29,30]$. For $H$. influenzae and M. catarrhalis qPCR assay, a cut-off of $>1 \times 10^{5}$ genomic copies $\cdot \mathrm{mL}^{-1}$ was considered $[31,32]$. The percentage of samples above the cut-off for S. pneumoniae, H. influenzae, M. catarrhalis and P. aeruginosa was $67.11 \%$ (51 out of 76), 71.05\% (54 out of 76 ), $65.79 \%$ (50 out of 76 ) and $47.47 \%$ (36 out of 76), respectively.

In H-NS subjects, the load of S. pneumoniae and H. influenzae in induced sputum samples was negligible compared with BMS-COPD and S-COPD subjects $(\mathrm{p}<0.0001)$ (figure $1 \mathrm{a}$ and $\mathrm{b}$ ). We observed that the load of S. pneumoniae and $H$. influenzae in induced sputum samples of BMS-COPD and S-COPD subjects was significantly higher compared with H-BMS (S. pneumoniae: $\mathrm{p}<0.0001$; H. influenzae: $\mathrm{p}=0.0002$ ) and HS (S. pneumoniae: $\mathrm{p}<0.0001 ; H$. influenzae: $\mathrm{p}=0.0002$ ) subjects (figure $1 \mathrm{a}$ and $\mathrm{b}$ ). Interestingly, in this study the load of $M$. catarrhalis in induced sputum was significantly higher in S-COPD subjects compared with BMS-COPD subjects $(\mathrm{p}<0.0001)$ (figure $1 \mathrm{c})$. However, the load of
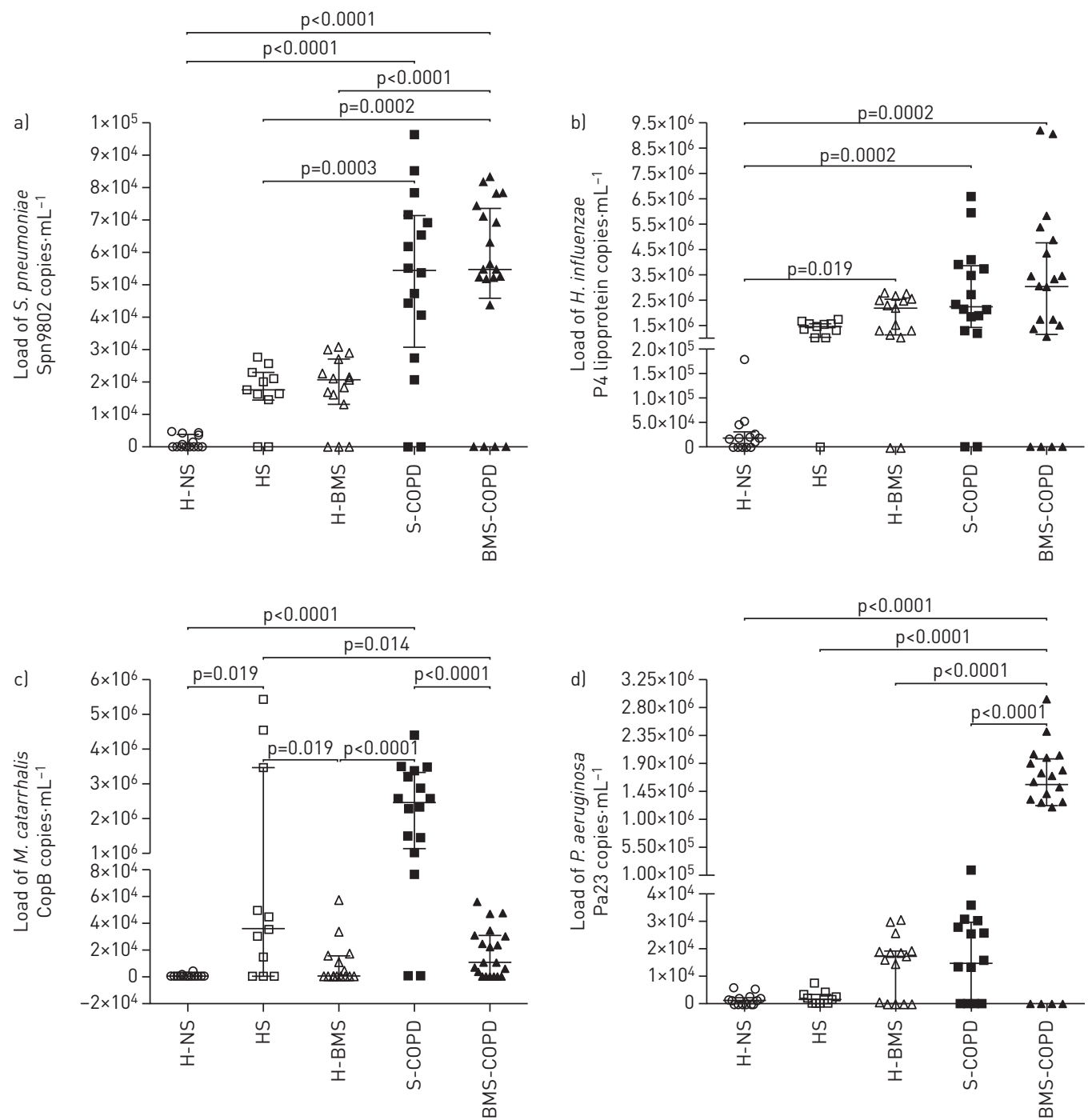

FIGURE 1 Bacterial load of potentially pathogenic bacteria in induced sputum samples among study subjects using quantitative PCR assay: a) Streptococcus pneumoniae, b) Haemophilus influenzae, c) Moraxella catarrhalis and d) Pseudomonas aeruginosa, in healthy nonsmokers (H-NS) (n=14), smokers without chronic obstructive pulmonary disease (COPD) (HS) ( $n=11)$, biomass smoke (BMS)-exposed healthy subjects (H-BMS) $(n=15)$, tobacco smoke-associated COPD subjects (S-COPD) ( $n=16)$ and BMS-exposed COPD (BMS-COPD) subjects $(n=20)$. The Shapiro-Wilk normality test was performed. Data are presented as dot plots with median (interquartile range). The Kruskal-Wallis test followed by Dunn's multiple comparisons test was performed for within-group comparisons. $p<0.05$ was considered statistically significant. 
$P$. aeruginosa was found to be higher in induced sputum samples of BMS-COPD subjects compared with S-COPD subjects $(\mathrm{p}<0.0001)$ (figure $1 \mathrm{~d})$.

A comparison of the PPB load in the airways among healthy controls was also performed. We did not observe any difference in the load of S. pneumoniae among healthy controls (H-NS, HS and H-BMS) (figure 1a). However, the load of $H$. influenzae in the airways of H-BMS and HS subjects was higher compared with H-NS subjects, but this load was found to be statistically significant only for H-BMS subjects $(\mathrm{p}=0.019)$ (figure $1 \mathrm{~b})$. Although the load of $M$. catarrhalis in healthy controls was below the cut-off limits, the load of $M$. catarrhalis in the airways of HS subjects was significantly elevated compared with H-NS and HS subjects ( $\mathrm{p}=0.019$ ) (figure 1c). Interestingly, $P$. aeruginosa showed an increased load in $\mathrm{H}$-BMS subjects compared with HS and H-NS subjects, but this load was not found to be statistically significant $(\mathrm{p}>0.05)$ (figure $1 \mathrm{~d})$.

\section{Load of pathogenic microbes as determined by microbial culturing}

In a subset of samples, we also investigated the PPB load through microbial culture methods, and confirmed S. pneumoniae and $H$. influenzae were significantly higher in the induced sputum samples of BMS-COPD and S-COPD subjects compared with healthy subjects $(\mathrm{p}<0.05)$ (supplementary figure S3a and b). Similar to the qPCR data, the CFU $\mathrm{mL}^{-1}$ values of $M$. catarrhalis were also higher in tobacco smoke-exposed subjects $(\mathrm{p}<0.05)$ (supplementary figure S3c), whereas $P$. aeruginosa was exclusively higher among BMS-exposed subjects $(\mathrm{p}<0.05)$ (supplementary figure $\mathrm{S} 3 \mathrm{~d})$.

In this study, the considered cut-off value for bacterial species to be pathogenic was $\geqslant 10^{4} \mathrm{CFU} \cdot \mathrm{mL}^{-1}$ for S. pneumoniae, $H$. influenzae and $M$. catarrhalis, and $\geqslant 10^{5} \mathrm{CFU} \cdot \mathrm{mL}^{-1}$ for P. aeruginosa [33]. The percentage of samples as determined by microbial culture methods above the cut-off for $S$. pneumoniae, $H$. influenzae, M. catarrhalis and P. aeruginosa was 52\% (13 out of 25), 80\% (20 out of 25), 40\% (10 out of 25$)$ and $40 \%$ (10 out of 25$)$, respectively.
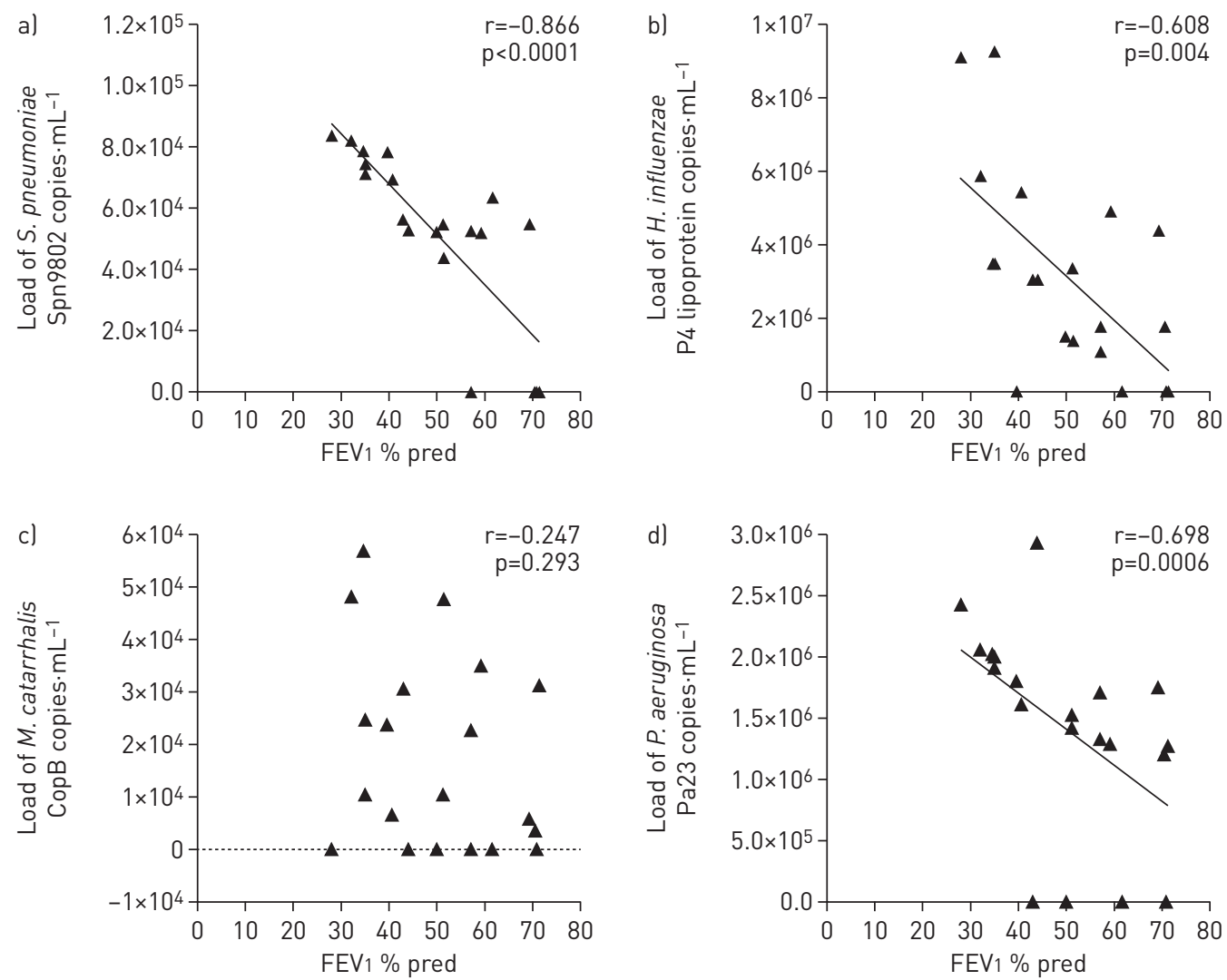

FIGURE 2 Relationship between bacterial load in induced sputum samples and forced expiratory volume in 1 s (FEV1) \% pred in biomass smoke-exposed chronic obstructive pulmonary disease subjects (n=20): a) Streptococcus pneumoniae, b) Haemophilus influenzae, c) Moraxella catarrhalis and d) Pseudomonas aeruginosa. The Shapiro-Wilk normality test was performed. Correlation coefficients were calculated using nonparametric Spearman correlation analysis. $\mathrm{p}<0.05$ was considered statistically significant. 
Correlation between bacterial load and lung function

Having shown differences in bacterial load in the sputum of subjects with COPD and controls, we wanted to determine whether these differences were associated with changes in lung function parameters.

In BMS-exposed subjects (BMS-COPD and H-BMS), the airway load of S. pneumoniae, H. influenzae and $P$. aeruginosa negatively correlated with $\mathrm{FEV}_{1} \%$ pred $(\mathrm{p}<0.05)$ (figure 2 and supplementary figure $\mathrm{S} 4$ ), FVC \% pred $(\mathrm{p}<0.05)$ (figure 3 and supplementary figure S5) and FEV1/FVC $(\mathrm{p}<0.05)$ (supplementary figures S6 and S7).

Similarly, in tobacco smoke-exposed subjects (S-COPD and HS) a significant negative correlation was observed between the load of S. pneumoniae, H. influenzae and $M$. catarrhalis and FEV1 \% pred $(\mathrm{p}<0.05)$ (supplementary figures S8 and S11), FVC \% pred ( $\mathrm{p}<0.05)$ (supplementary figures S9 and S12) and FEV1/ FVC $(\mathrm{p}<0.05)$ (supplementary figures S10 and S13).

\section{Correlation between bacterial load and age, smoking history and biomass exposure history}

With a multivariate linear regression model, we showed that age was not associated with PPB load among the subjects $(\mathrm{p}>0.05)$ (supplementary table S4), and that BMS exposure history was statistically positively associated with the load of $S$. pneumoniae, H. influenzae and $P$. aeruginosa $(\mathrm{p}<0.05)$ (supplementary table S4). However, tobacco smoking history was significantly positively associated with the load of $S$. pneumoniae, H. influenzae and M. catarrhalis $(\mathrm{p}<0.05)$ (supplementary table $\mathrm{S} 4)$.

\section{Comparison of total sputum macrophages among the subject groups}

The total count of sputum macrophages was also investigated. We observed that the number of macrophages present in the induced sputum samples of BMS-COPD subjects was higher compared with $\mathrm{H}-\mathrm{BMS}$ and H-NS subjects, but was statistically significant compared with H-NS subjects $(\mathrm{p}=0.0005)$ (supplementary figure S14). Similarly, in S-COPD subjects, the number of sputum macrophages was found
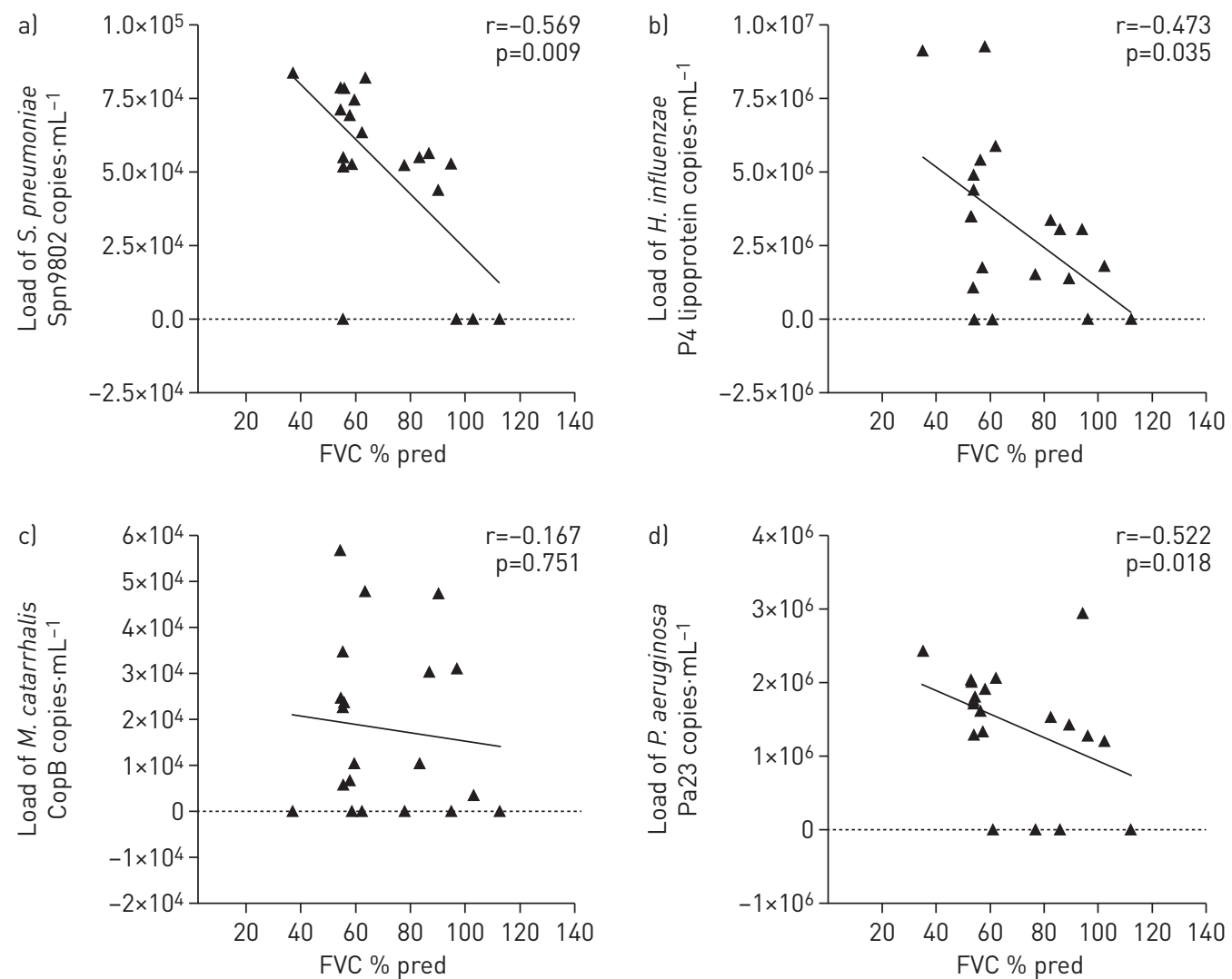

FIGURE 3 Relationship between bacterial load in induced sputum samples and lung forced vital capacity (FVC) $\%$ pred in biomass smoke-exposed chronic obstructive pulmonary disease subjects $(n=20)$ : a) Streptococcus pneumoniae, b) Haemophilus influenzae, c) Moraxella catarrhalis and d) Pseudomonas aeruginosa. The ShapiroWilk normality test was performed. Correlation coefficients were calculated using nonparametric Spearman correlation analysis. $p<0.05$ was considered statistically significant. 
to be significantly higher compared with HS ( $\mathrm{p}=0.0004)$ and H-NS $(\mathrm{p}<0.0001)$ subjects (supplementary figure S14).

\section{Correlation between total sputum macrophages and bacterial load in airways}

We analysed the association between total sputum macrophage count and PPB load in the airways. A significant positive association was observed between total sputum macrophages and the load of S. pneumoniae and H. influenzae in BMS-COPD and S-COPD subjects $(\mathrm{p}<0.05)$ (supplementary figures S15 and S17). Interestingly, the load of $P$. aeruginosa was found to be positively associated with total sputum macrophages in BMS-exposed subjects (BMS-COPD and H-BMS) $(p<0.05)$ (supplementary figures S15 and S16), whereas the load of $M$. catarrhalis was associated with total sputum macrophages in tobacco smoke-exposed subjects (S-COPD and HS) ( $\mathrm{p}<0.05)$ (supplementary figures S17 and S18).

\section{Correlation between bacterial load and quality of life scores of COPD subjects}

We also investigated the relationship between the colonisation of these PPB in the airways and quality of life in COPD. In BMS-COPD subjects, a significant positive association was observed between the total SGRQ score and the load of $S$. pneumoniae ( $\mathrm{r}=0.692, \mathrm{p}=0.0007), H$. influenzae $(\mathrm{r}=0.579, \mathrm{p}=0.007)$ and $P$. aeruginosa $(\mathrm{r}=0.640, \mathrm{p}=0.002)$ (figure 4). Similarly, a significant positive association was observed between the total CAT score and the load of $S$. pneumoniae $(\mathrm{r}=0.564, \mathrm{p}=0.009)$, H. influenzae $(\mathrm{r}=0.614$, $\mathrm{p}=0.004)$ and $P$. aeruginosa $(\mathrm{r}=0.792, \mathrm{p}<0.0001)$, but not $M$. catarrhalis (figure 5).

A significant positive association in S-COPD subjects was observed between the total SGRQ and CAT scores and the load of $S$. pneumoniae, $H$. influenzae and $M$. catarhalis, but not $P$. aeruginosa $(\mathrm{p}<0.05)$ (supplementary figures S19 and S20).

\section{Phagocytic activity of MDMs}

Having established increased airway bacterial load in COPD subjects, we examined whether this defect could be associated with reduced innate immune responses such as decreased phagocytosis. We observed
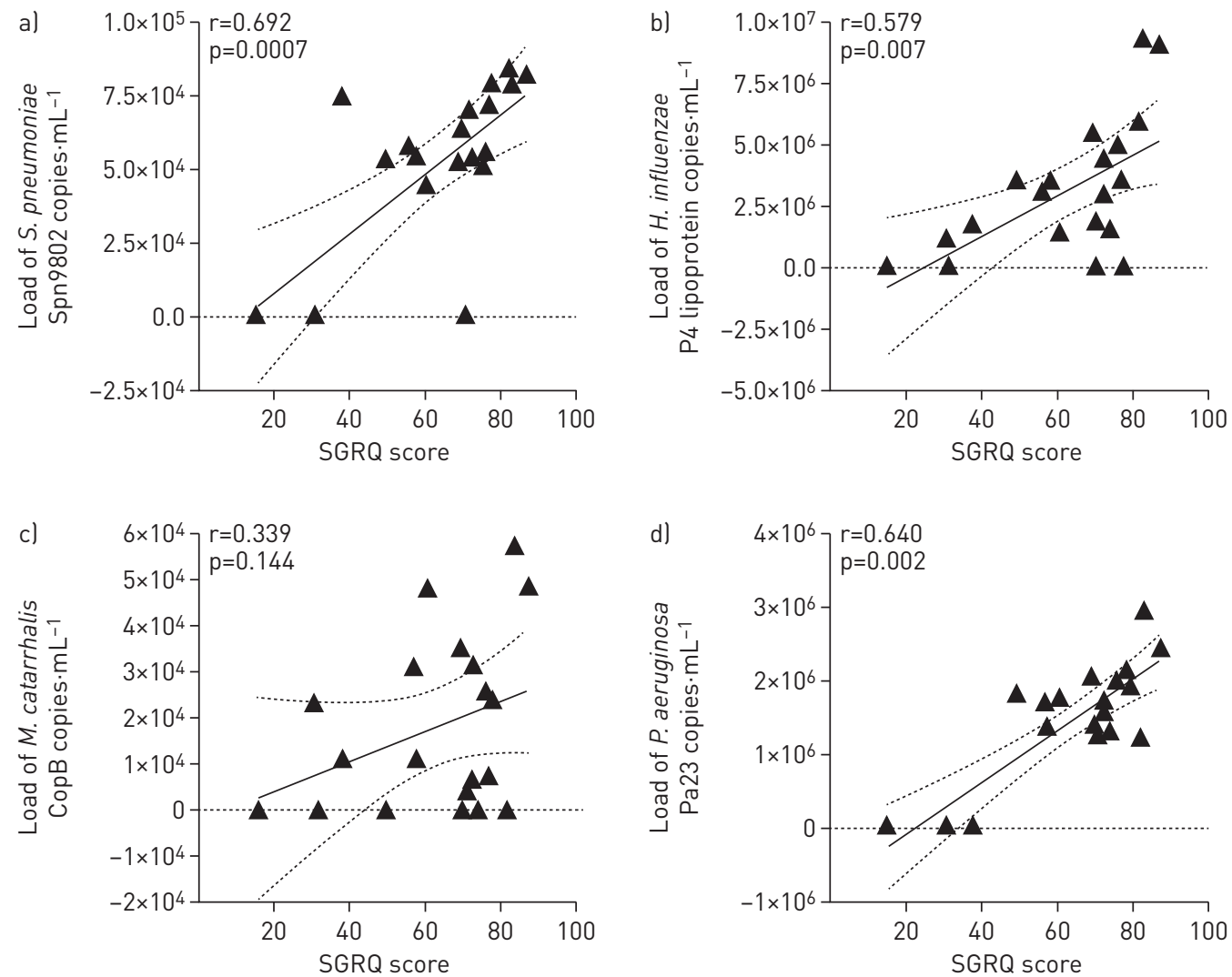

FIGURE 4 Relationship between bacterial load in induced sputum samples and total St George's Respiratory Questionnaire (SGRQ) score in biomass smoke-exposed chronic obstructive pulmonary disease subjects $(n=20)$ : a) Streptococcus pneumoniae, b) Haemophilus influenzae, c) Moraxella catarrhalis and d) Pseudomonas aeruginosa. The Shapiro-Wilk normality test was performed. Correlation coefficients were calculated using nonparametric Spearman correlation analysis. $p<0.05$ was considered statistically significant. 

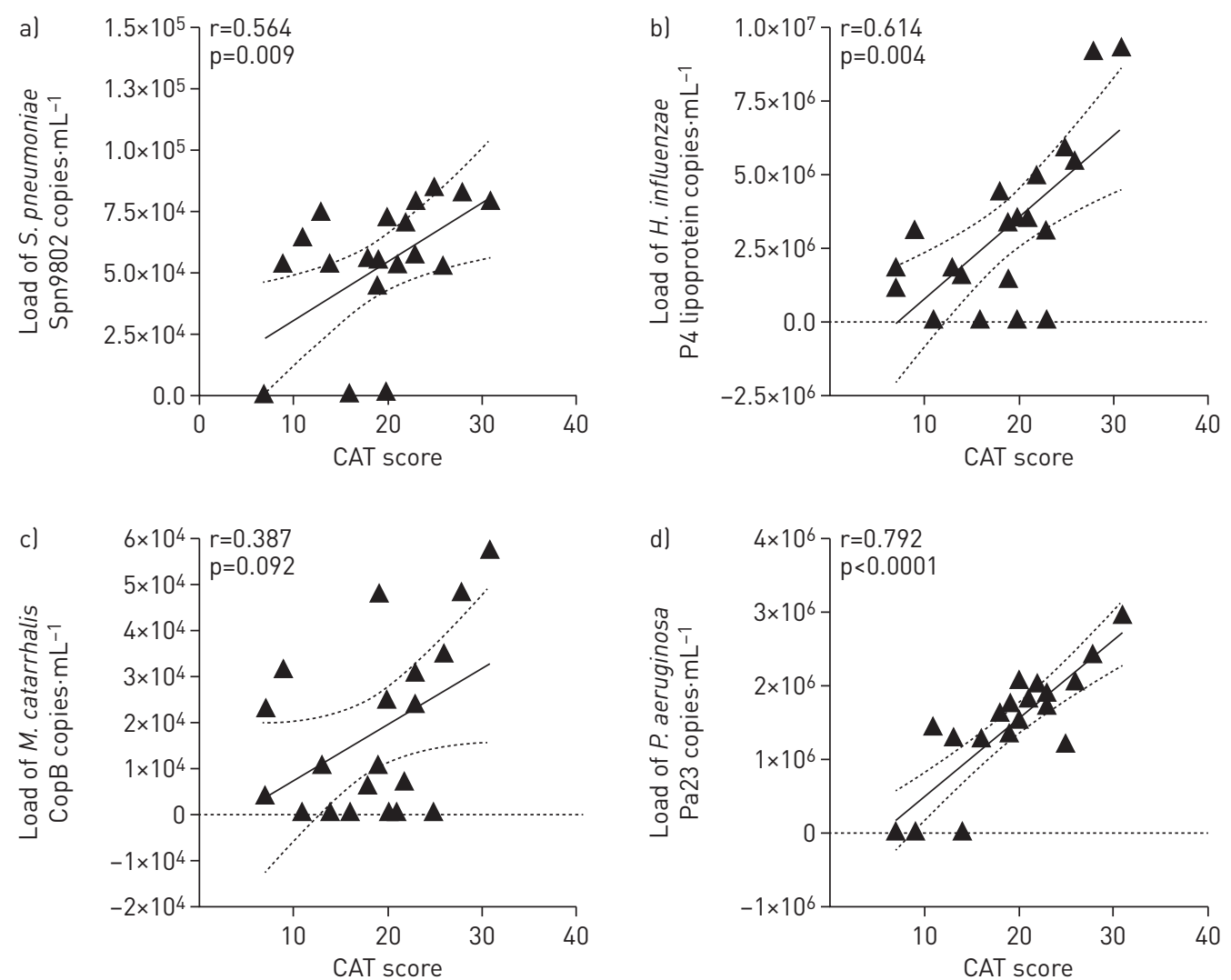

FIGURE 5 Relationship between bacterial load in induced sputum samples and total chronic obstructive pulmonary disease (COPD) Assessment Test (CAT) score in biomass smoke-exposed COPD subjects $(n=20)$ : a) Streptococcus pneumoniae, b) Haemophilus influenzae, c) Moraxella catarrhalis and d) Pseudomonas aeruginosa. The Shapiro-Wilk normality test was performed. Correlation coefficients were calculated using nonparametric Spearman correlation analysis. $p<0.05$ was considered statistically significant.

no differences in the phagocytosis of inert beads by MDMs between the different subject groups $(\mathrm{p}>0.05)$ (figure 6a), implying that all MDMs were capable of phagocytosis. We also compared bacterial phagocytic activity of MDMs among male (H-NS, HS, S-COPD) and female (H-BMS and BMS-COPD) subjects; however, we did not observe any significant difference in the MDM phagocytic activity of each PPB and overall phagocytic activity (mean phagocytic activity of beads, S. pneumoniae and H. influenzae) between male and female subjects ( $\mathrm{p}>0.05)$ (supplementary figure S21).

We next examined the response of these MDMs to fluorescently tagged heat-killed PPB (S. pneumoniae and $H$. influenzae), and observed impaired phagocytosis by MDMs of BMS-COPD and S-COPD subjects compared with H-NS subjects (S. pneumoniae and $H$. influenzae: $\mathrm{p}<0.0001$ ) (figure $6 \mathrm{~b}$ and $\mathrm{c}$ ). Also, MDMs from BMS-COPD subjects demonstrated significantly impaired bacterial phagocytosis compared with H-BMS subjects (S. pneumoniae: $\mathrm{p}=0.0001 ; H$. influenzae: $\mathrm{p}=0.031$ ) (figure $6 \mathrm{~b}$ and $\mathrm{c}$ ). Similarly, MDMs from S-COPD subjects demonstrated impaired phagocytic activity compared with HS subjects, but this difference was found to be not statistically significant (S. pneumoniae and $H$. influenzae: $\mathrm{p}>0.05$ ) (figure $6 \mathrm{~b}$ and $\mathrm{c}$ ).

In addition, we observed that MDMs from H-NS subjects exhibited a significantly higher PPB phagocytic activity compared with HS subjects ( $S$. pneumoniae: $\mathrm{p}=0.012 ; \mathrm{H}$. influenzae: $\mathrm{p}=0.015)$, but not compared with $\mathrm{H}$-BMS subjects ( $S$. pneumoniae and $H$. influenzae: $\mathrm{p}>0.05$ ) (figure $6 \mathrm{~b}$ and $\mathrm{c}$ ). Interestingly, there were no significant differences in the MDM phagocytic activity for $S$. pneumoniae and $H$. influenzae within COPD (BMS-COPD and S-COPD) and healthy exposed (H-BMS and HS) subjects ( $>0.05)$ (figure $6 \mathrm{~b}$ and $\mathrm{c}$ ).

Relationship between bacterial phagocytosis by MDMs and induced sputum bacterial load Having identified a defect in innate immune responses, we wanted to determine whether this could be related to PPB load in the airways of these subjects. We observed that phagocytic activity by MDMs from BMS-exposed subjects (BMS-COPD and H-BMS) for both S. pneumoniae and H. influenzae negatively 

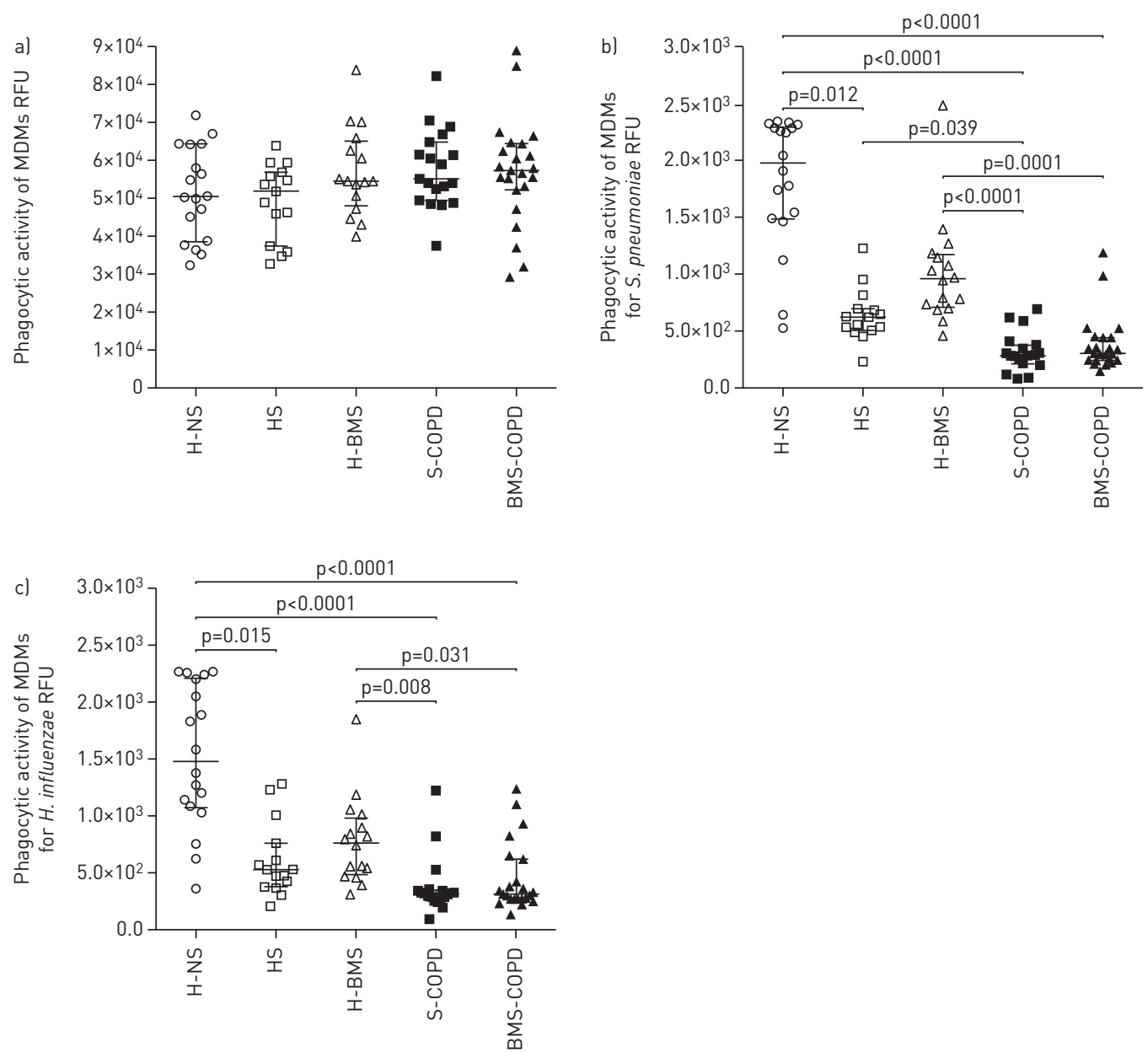

FIGURE 6 Phagocytosis of fluorescently labelled beads and bacteria by monocyte-derived macrophages (MDMs): a) Streptococcus pneumoniae, b) Haemophilus influenzae, c) Moraxella catarrhalis and d) Pseudomonas aeruginosa, in healthy nonsmokers ( $H-N S)(n=18)$, smokers without chronic obstructive pulmonary disease (COPD) (HS) ( $n=15)$, biomass smoke (BMS)-exposed healthy subjects (H-BMS) ( $n=16)$, tobacco smoke-associated COPD subjects (S-COPD) $(n=19)$ and BMS-exposed COPD (BMS-COPD) subjects $(n=23)$. RFU: relative fluorescence units. The Shapiro-Wilk normality test was performed. Data are presented as dot plots with median (interquartile range). The Kruskal-Wallis test followed by Dunn's multiple comparisons test was performed for within-group comparisons. $p<0.05$ was considered statistically significant.

correlated with induced sputum bacterial load (BMS-COPD: S. pneumoniae: $\mathrm{r}=-0.752, \mathrm{p}=0.0001$ and H. influenzae: $\mathrm{r}=-0.743, \mathrm{p}=0.0002$; H-BMS: $S$. pneumoniae: $\mathrm{r}=-0.699, \mathrm{p}=0.005$ and $H$. influenzae: $\mathrm{r}=-0.556$, $\mathrm{p}=0.034$ ) (figure 7 and supplementary figure S23). Similarly, phagocytic activity of MDMs from tobacco smoke-exposed subjects (S-COPD and HS) negatively correlated with increased load of S. pneumoniae and H. influenzae in induced sputum (supplementary figures S24 and S25).

Relationship between bacterial phagocytosis by MDMs and spirometric lung function indices

Having shown differences in PPB phagocytic activity by MDMs and its association with bacterial load in the airways, we wanted to determine if these differences were associated with changes in lung function parameters. We observed that in both BMS- and tobacco smoke-exposed subjects, MDM phagocytosis of $S$. pneumoniae and $H$. influenzae was positively associated with spirometric lung function indices $(\mathrm{p}<0.05)$ (supplementary figures S26-S37).

\section{Relationship between bacterial phagocytosis by MDMs and demographic characteristics}

We also investigated whether there was any relationship between bacterial phagocytic activity of MDMs and demographic characteristics. We found that age was not associated with MDM phagocytic activity across all subjects $(\mathrm{p}>0.05)$ (supplementary table S5). Both BMS and tobacco smoke exposure history were strongly positively associated with MDM phagocytosis of $S$. pneumoniae and $H$. influenzae $(\mathrm{p}<0.05)$ (supplementary table S5). 

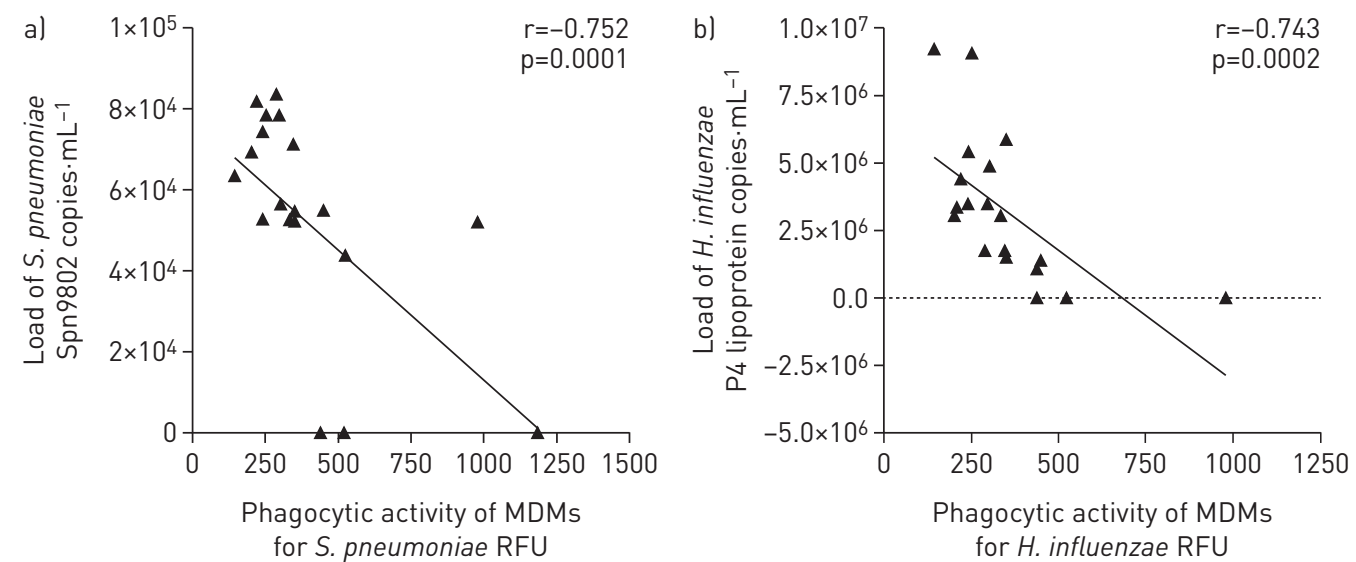

FIGURE 7 Relationship between bacterial load in induced sputum samples and phagocytic activity of monocyte-derived macrophages (MDMs) in biomass smoke-exposed chronic obstructive pulmonary disease (COPD) subjects ( $n=20)$ : a) Streptococcus pneumoniae and b) Haemophilus influenzae. RFU: relative fluorescence units. The Shapiro-Wilk normality test was performed. Correlation coefficients were calculated using Spearman correlation analysis. $p<0.05$ was considered statistically significant.

Relationship between bacterial phagocytosis by MDMs and quality of life of COPD subjects We investigated the relationship between MDM phagocytic activity for PPB and the quality of life of COPD subjects. For BMS-COPD subjects, there was a significant negative association between the total CAT score and MDM phagocytosis of $S$. pneumoniae $(\mathrm{r}=-0.444, \mathrm{p}=0.034)$ and $H$. influenzae $(\mathrm{r}=-0.416$, $\mathrm{p}=0.048$ ) (supplementary figure S38). A similar significant negative association was observed between the total SGRQ score and MDM phagocytosis of $S$. pneumoniae $(\mathrm{r}=-0.453, \mathrm{p}=0.029)$ and $H$. influenzae $(\mathrm{r}=0.579, \mathrm{p}=0.007)$ (supplementary figure S40).

A similar relationship was also observed for S-COPD subjects, where there appeared to be a significant negative association between the total CAT and SGRQ scores and the phagocytic activity of MDMs for $S$. pneumoniae and H. influenzae (supplementary figures S39 and S41).

\section{Viability assay}

It was possible that the reduced phagocytic responses observed could be due to bacteria-induced cell death. In order to investigate this, MTT assays to measure cell viability were performed. Throughout the experimental conditions the viability of MDMs was $\geqslant 90 \%$ for all samples (figure 8 ).

\section{Discussion}

Macrophages are key protective innate immune cells that guard against invasion of PPB. S-COPD subjects harbour an increased PPB load (e.g. S. pneumoniae, H. influenzae, M. catarrhalis and P. aeruginosa) in the lower airways, which is likely to contribute to recurrent infections in the respiratory tract $[7,9,11]$. The burden of these PPB in the respiratory tract of S-COPD subjects was found to be associated with decline in lung function indices and impairment in clearance of PPB by alveolar macrophages, which is reflected in the response of MDMs as well $[14,15,17,22]$. In this study, we also observed that the respiratory tracts of S-COPD subjects from a rural Indian population harbour similar PPB, which are associated with defective phagocytic activity of MDMs and decline in spirometric lung function indices.

BMS has been suggested to be greater risk factor for COPD globally than tobacco smoke because of the sheer numbers of people exposed to BMS [5]. It is possible that long-term exposure to BMS is associated with a similar defect in the innate immunity as observed in S-COPD subjects. We report here for the first time in BMS-COPD subjects a similar association of PPB colonisation in the respiratory tract with the defective phagocytic activity of MDMs and a reduction in lung function.

We found that the bacterial loads of $S$. pneumoniae and $H$. influenzae in sputum samples were similar in both BMS-COPD and S-COPD subjects. Consequently, the phagocytic activity of MDMs from BMS-COPD subjects was investigated and compared with healthy controls (H-NS, HS and H-BMS) and S-COPD subjects. The phagocytic activity of MDMs from BMS-COPD subjects was not significantly altered compared with S-COPD subjects, but was much reduced compared with healthy controls. This suggests that similar mechanisms relating to defects in the innate immune response may apply in both BMS-COPD and S-COPD subjects. There is no doubt that BMS increases particular uptake in alveolar macrophages and this may contribute to defective phagocytosis [34-36]. However, we report reduced 

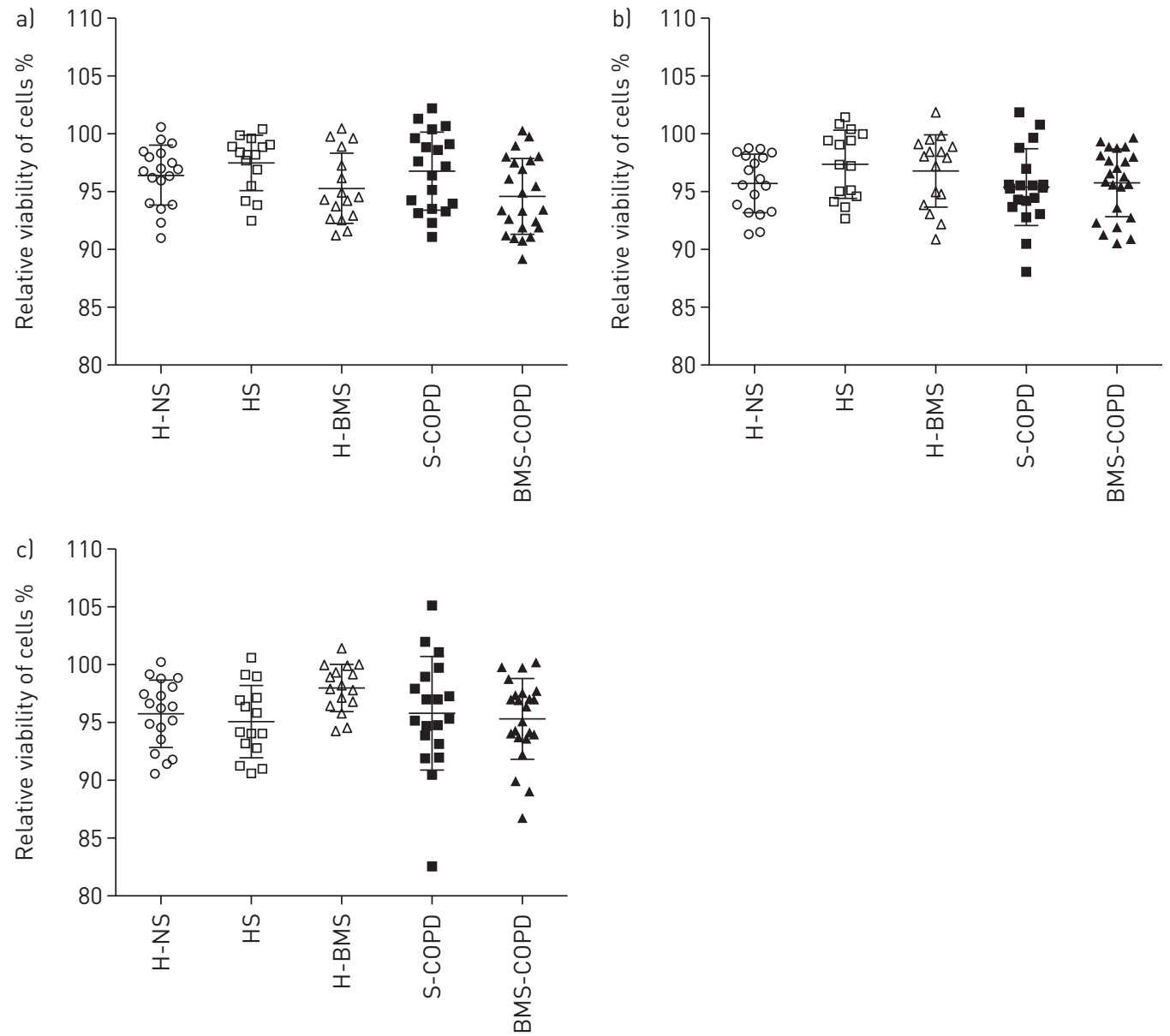

FIGURE 8 Effect of fluorescently labelled beads and bacteria on monocyte-derived macrophage (MDM) viability: a) fluorescently labelled beads, b) Streptococcus pneumoniae and c) Haemophilus influenzae, in healthy nonsmokers (H-NS) ( $n=18)$, smokers without chronic obstructive pulmonary disease (COPD) (HS) $(n=15)$, biomass smoke (BMS)-exposed healthy subjects (H-BMS) $(n=16)$, tobacco smoke-associated COPD subjects (S-COPD) ( $n=19$ ) and BMS-exposed COPD (BMS-COPD) subjects ( $n=23$ ). The Shapiro-Wilk normality test was performed. Data are presented as dot plots with median (interquartile range). Ordinary one-way ANOVA followed by Tukey's multiple comparisons test was performed. $p<0.05$ was considered statistically significant.

phagocytosis by MDMs that had not been exposed to these particulates and suggest that there is a common defective mechanism inherent in the circulating cells.

Prior animal and human studies have reported that inhaled noxious pollutants translocate from the lungs to the systemic circulation [37], suggesting a direct link between the lungs and the systemic circulation. Exposure to noxious pollutants present in smoke can possibly contribute to an excessive inflammatory response of the lungs that spills over from the pulmonary compartment and contributes to systemic inflammation [38]. It has been reported that decreased Nrf2 (nuclear factor erythroid 2-related factor 2) signalling in alveolar macrophages is linked to altered phagocytic activity and bacterial clearance in these cells in COPD subjects, and that the Nrf2 activator sulforaphane improves this response [39, 40]. Further research is needed to explore Nrf2 signalling mechanisms and the role of BMS constituents in the phagocytic activity of innate immune cells.

In Western populations, M. catarrhalis is found in the sputum samples of S-COPD subjects for shorter durations and is considered to be one of the major contributors to exacerbations [41]. P. aeruginosa was also reported in some stable S-COPD subjects and was associated with disease severity [41]. In our study, none of the S-COPD subjects were sampled during an exacerbation and yet colonisation of M. catarrhalis was observed. Interestingly, we observed that BMS-COPD and H-BMS subjects harbour more $P$. aeruginosa, whereas S-COPD and HS subjects harbour more M. catarrhalis. As reported previously, exposure to BMS contributes to an altered bacterial community [42]. In our study, we also observed that the increased colonisation of $\mathrm{PPB}$ in the airways and impaired MDM phagocytic activity worsened the 
quality of life (SGRQ and CAT scores) in BMS-COPD subjects. Further investigation is required to understand the underlying mechanisms associated with $P$. aeruginosa and $M$. catarrhalis colonisation and the phagocytic activity of macrophages.

Our study has some limitations. First, this study involved identifying pathogenic bacteria in induced sputum samples. Upon sample collection, there is a possibility that the induced samples might be contaminated with the upper respiratory tract or oral microbial flora. However, there is evidence to suggest that induced sputum is the most sensitive, qualitative and quantitative method for diagnosing an infective obstructive airway diseases [43]. Second, our study was sex biased, i.e. H-NS, HS and S-COPD subjects were all male, whereas H-BMS and BMS-COPD subjects were all female. However, we did not observe any difference in PPB load and phagocytic activity of MDMs among male and female subjects. It is unlikely that sex directly impacts on macrophage phagocytic activity, risk of developing COPD and colonisation with PPB. This was a real-world scenario, where the female Indian population is primarily involved in cooking and is exposed to BMS. We did make an attempt to minimise this bias by correcting the spirometric indices for sex and performing analyses using percentage predicted values. Also, we did not observe any sex-specific difference in the phagocytic activity of the Western COPD subjects (supplementary figure S22). Additionally, prior studies have reported that colonisation of $M$. catarrhalis in airways is not associated with sex [44]. Third, to evaluate the phagocytic activity of MDMs, we used nonopsonised S. pneumoniae and $H$. influenzae, which indicates that activity of opsonised receptors of MDMs was limited in this study. Phagocytosis of opsonised bacteria is also impaired in COPD in both alveolar macrophages and MDMs [40]. In the lung, the role of opsonisation driving phagocytosis is limited as it is not considered a serum-rich environment. Nevertheless, opsonisation of surfactant proteins and other lung-derived immunoglobulins is possible.

In conclusion, we have shown for the first time that subjects with nonsmoking COPD are similar to those with S-COPD, as they harbour PPB in their airways that are associated with a defect in macrophage phagocytosis. This defect in macrophages is possibly associated with increased susceptibility of those exposed to noxious gases to develop airflow obstruction and this is a common susceptibility factor in tobacco smoke or BMS exposure.

Acknowledgements: We would like to thank the patients who had participated in this study. We would like to thank Meena Raykar and Savita Walke (Vadu Rural Health Program, KEM Hospital Research Center, Pune, India) for their support in patient recruitment.

Author contributions: J.D. Londhe was involved in patient recruitment from the CRF-COPD cohort. CRF-COPD cohort establishment was supervised by S. Juvekar and S.S. Salvi. V.V. Das performed lung function tests on the recruited subjects. B. Brashier established the diagnosis of subjects. B. Ghosh and A.H. Gaike performed qPCR and bacterial culture assays under the supervision of Y.S. Shouche. K. Pyasi was involved the collection of sputum and blood samples from the recruited subjects. K. Pyasi also performed differential cell counts on the induced sputum samples. B. Ghosh was trained by L.E. Donnelly for generation of MDMs and the phagocytic activity assays. B. Ghosh and K. Pyasi performed phagocytic assays. B. Ghosh performed statistical analysis and wrote the manuscript under the supervision of S.S. Salvi. P.J. Barnes and S.S. Salvi were the principal investigators of this study and conceived the idea for the study.

Conflict of interest: B. Ghosh has nothing to disclose. A.H. Gaike has nothing to disclose. K. Pyasi has nothing to disclose. B. Brashier has nothing to disclose. V.V. Das has nothing to disclose. J.D. Londhe has nothing to disclose. S. Juvekar has nothing to disclose. Y.S. Shouche has nothing to disclose. L.E. Donnelly reports grants from Cempra, AstraZeneca and Boehringer Ingelheim, outside the submitted work. S.S. Salvi has nothing to disclose. P.J. Barnes has nothing to disclose.

Support statement: This study was funded by Indian Council of Medical Research (India) and Medical Research Council (UK). Funding information for this article has been deposited with the Crossref Funder Registry.

\section{References}

1 Lozano R, Naghavi M, Foreman K, et al. Global and regional mortality from 235 causes of death for 20 age groups in 1990 and 2010: a systematic analysis for the Global Burden of Disease Study 2010. Lancet 2012; 380: 2095-2128.

2 Nongkynrih B, Patro BK, Pandav CS. Current status of communicable and non-communicable diseases in India. J Assoc Physicians India 2004; 52: 118-123.

3 Lundback B, Lindberg A, Lindstrom M, et al. Not 15 but 50\% of smokers develop COPD? Report from the Obstructive Lung Disease in Northern Sweden Studies. Respir Med 2003; 97: 115-122.

4 Burney $\mathrm{P}$, Jithoo A, Kato B, et al. Chronic obstructive pulmonary disease mortality and prevalence: the associations with smoking and poverty — a BOLD analysis. Thorax 2014; 69: 465-473.

5 Salvi SS, Barnes PJ. Chronic obstructive pulmonary disease in non-smokers. Lancet 2009; 374: $733-743$.

6 Wu D, Hou C, Li Y, et al. Analysis of the bacterial community in chronic obstructive pulmonary disease sputum samples by denaturing gradient gel electrophoresis and real-time PCR. BMC Pulm Med 2014; 14: 1-7.

7 Curran T, Coyle PV, McManus TE, et al. Evaluation of real-time PCR for the detection and quantification of bacteria in chronic obstructive pulmonary disease. FEMS Immunol Med Microbiol 2007; 50: 112-118.

8 Barker BL, Haldar K, Patel H, et al. Association between pathogens detected using quantitative polymerase chain reaction with airway inflammation in COPD at stable state and exacerbations. Chest 2015; 147: 46-55. 
9 Wang $\mathrm{H}, \mathrm{Gu} \mathrm{X}$, Weng $\mathrm{Y}$, et al. Quantitative analysis of pathogens in the lower respiratory tract of patients with chronic obstructive pulmonary disease. BMC Pulm Med 2015; 15: 94.

10 Rennard SI. Exacerbations and progression of disease in asthma and chronic obstructive pulmonary disease. Proc Am Thorac Soc 2004; 1: 88-92.

11 Patel IS, Seemungal TAR, Wilks M, et al. Relationship between bacterial colonisation and the frequency, character, and severity of COPD exacerbations. Thorax 2002; 57: 759-764.

12 Donnelly LE, Barnes PJ. Defective phagocytosis in airways disease. Chest 2012; 141: 1055-1062.

13 Berenson CS, Kruzel RL, Eberhardt E, et al. Phagocytic dysfunction of human alveolar macrophages and severity of chronic obstructive pulmonary disease. J Infect Dis 2013; 208: 2036-2045.

14 Berenson CS, Kruzel RL, Eberhardt E, et al. Impaired innate immune alveolar macrophage response and the predilection for COPD exacerbations. Thorax 2014; 69: 811-818.

15 Garcha DS, Thurston SJ, Patel ARC, et al. Changes in prevalence and load of airway bacteria using quantitative PCR in stable and exacerbated COPD. Thorax 2012; 67: 1075-1080.

16 Barnes PJ. Chronic obstructive pulmonary disease. N Engl J Med 2000; 343: 269-280.

17 Taylor AE, Finney-Hayward TK, Quint JK, et al. Defective macrophage phagocytosis of bacteria in COPD. Eur Respir J 2010; 35: 1039-1047.

18 Liang Z, Zhang Q, Thomas CM, et al. Impaired macrophage phagocytosis of bacteria in severe asthma. Respir Res 2014; 15: 72 .

19 Paemka L, McCullagh BN, Abou Alaiwa MH, et al. Monocyte derived macrophages from CF pigs exhibit increased inflammatory responses at birth. J Cyst Fibros 2017; 16: 471-474.

20 Rylance J, Fullerton DG, Scriven J, et al. Household air pollution causes dose-dependent inflammation and altered phagocytosis in human macrophages. Am J Respir Cell Mol Biol 2014; 52: 584-593.

21 Morales-Nebreda L, Misharin AV, Perlman H, et al. The heterogeneity of lung macrophages in the susceptibility to disease. Eur Respir Rev 2015; 24: 505-509.

22 Hodge S, Reynolds PN. Low-dose azithromycin improves phagocytosis of bacteria by both alveolar and monocyte-derived macrophages in chronic obstructive pulmonary disease subjects. Respirology 2012; 17: 802-807.

23 Jones PW, Harding G, Berry P, et al. Development and first validation of the COPD Assessment Test. Eur Respir J 2009; 34: 648-654.

24 Meguro M, Barley EA, Spencer S, et al. Development and validation of an improved, COPD-specific version of the St. George respiratory questionnaire. Chest 2007; 132: 456-463.

25 Global Initiative for Chronic Obstructive Lung Disease. Global Strategy for the Diagnosis, Management, and Prevention of Chronic Obstructive Pulmonary Disease. 2017. http://goldcopd.org/gold-2017-global-strategy-diagnosismanagement-prevention-copd Date last accessed: December 5, 2018.

26 Golpe R, Martín-Robles I, Sanjuán-López P, et al. Differences in systemic inflammation between cigarette and biomass smoke-induced COPD. Int J Chron Obstruct Pulmon Dis 2017; 12: 2639-2646.

27 Miller MR, Hankinson J, Brusasco V, et al. Standardisation of spirometry. Eur Respir J 2005; 26: 319-338.

28 McCulloch E, Lucas C, Ramage G, et al. Improved early diagnosis of Pseudomonas aeruginosa by real-time PCR to prevent chronic colonisation in a paediatric cystic fibrosis population. J Cyst Fibros 2011; 10: 21-24.

29 Abdeldaim GMK, Strålin K, Olcén P, et al. Toward a quantitative DNA-based definition of pneumococcal pneumonia: a comparison of Streptococcus pneumoniae target genes, with special reference to the Spn9802 fragment. Diagn Microbiol Infect Dis 2008; 60: 143-150.

30 Wang $\mathrm{K}, \mathrm{Xi} \mathrm{W}$, Yang $\mathrm{D}$, et al. Rhinovirus is associated with severe adult community-acquired pneumonia in China. J Thorac Dis 2017; 9: 4502-4511.

31 Abdeldaim GMK, Stralin K, Korsgaard J, et al. Multiplex quantitative PCR for detection of lower respiratory tract infection and meningitis caused by Streptococcus pneumoniae, Haemophilus influenzae and Neisseria meningitidis. BMC Microbiol 2010; 10: 310

32 Park DE, Baggett HC, Howie SRC, et al. Colonization density of the upper respiratory tract as a predictor of pneumonia - Haemophilus influenzae, Moraxella catarrhalis, Staphylococcus aureus, and Pneumocystis jirovecii. Clin Infect Dis 2017; 64: S328-S336.

33 Campbell S, Forbes BA. The clinical microbiology laboratory in the diagnosis of lower respiratory tract infections. J Clin Microbiol 2011; 49: 30-33.

34 Kulkarni NS, Prudon B, Panditi SL, et al. Carbon loading of alveolar macrophages in adults and children exposed to biomass smoke particles. Sci Total Environ 2005; 345: 23-30.

35 Fullerton DG, Jere K, Jambo K, et al. Domestic smoke exposure is associated with alveolar macrophage particulate load. Trop Med Int Health 2009; 14: 349-354.

36 Rylance J, Chimpini C, Semple S, et al. Chronic household air pollution exposure is associated with impaired alveolar macrophage function in Malawian non-smokers. PLoS One 2015; 10: 1-15.

37 Nemmar A, Hoylaerts MF, Hoet PHM, et al. Possible mechanisms of the cardiovascular effects of inhaled particles: systemic translocation and prothrombotic effects. Toxicol Lett 2004; 149: 243-253.

38 Agustí A. Systemic effects of chronic obstructive pulmonary disease: what we know and what we don't know (but should). Proc Am Thorac Soc 2007; 4: 522-525.

39 Harvey CJ, Thimmulappa RK, Sethi S, et al. Targeting Nrf2 signaling improves bacterial clearance by alveolar macrophages in patients with COPD and in a mouse model. Sci Transl Med 2011; 3: 78ra32.

40 Bewley MA, Budd RC, Ryan E, et al. Opsonic phagocytosis in chronic obstructive pulmonary disease is enhanced by Nrf2 agonists. Am J Respir Crit Care Med 2018; 198: 739-750.

41 Murphy TF, Brauer AL, Grant BJB, et al. Moraxella catarrhalis in chronic obstructive pulmonary disease: burden of disease and immune response. Am J Respir Crit Care Med 2005; 172: 195-199.

42 Rylance J, Kankwatira A, Nelson DE, et al. Household air pollution and the lung microbiome of healthy adults in Malawi: a cross-sectional study. BMC Microbiol 2016; 16: 1-7.

43 Gupta KB, Garg S. Sputum induction - a useful tool in diagnosis of respiratory diseases. Lung India 2006; 23 : 82-86.

44 Pettigrew MM, Gent JF, Revai K, et al. Microbial interactions during upper respiratory tract infections. Emerging Infect Dis 2008; 14: 1584-1591. 